\title{
SAÚDE EM COMUNIDADE QUILOMBOLA: CARACTERIZAÇÃO AMBIENTAL E OCORRÊNCIA DE ENTEROPARASITOSES
}

\author{
Hildebrando CIRQUEIRA JÚNIOR ${ }^{1}$ \\ Soraya de Matos Camargo GROSSMANN ${ }^{2}$ \\ Nadja Maria Gomes MURTA ${ }^{3}$ \\ João Victor Leite DIAS ${ }^{4}$ \\ Herton Helder Rocha PIRES ${ }^{5}$
}

\author{
${ }^{1}$ Universidade Vale do Rio Verde (UNINCOR). juniordiamantina@ hotmail.com \\ ${ }^{2}$ Universidade Vale do Rio Verde (UNINCOR). prof.soraya.grossmann@unincor.edu.br \\ ${ }^{3}$ Universidade Federal dos Vales do Jequitinhonha e Mucuri (UFVJM). nadjamurta@gmail.com \\ ${ }^{4}$ Universidade Federal dos Vales do Jequitinhonha e Mucuri (UFVJM). Centro de Pesquisas René Rachou - FIOCRUZ. \\ jvigento@yahoo.com.br \\ Universidade Federal dos Vales do Jequitinhonha e Mucuri (UFVJM). herton.pires@ufvjm.edu.br
}

\section{Recebido em: 24/08/2015 - Aprovado em: 11/11/2015 - Disponibilizado em: 18/12//2015}

\begin{abstract}
RESUMO: O objetivo do presente trabalho foi descrever a estrutura domiciliar e a ocorrência de enteroparasitoses em uma comunidade quilombola do município de Diamantina, Minas Gerais. Foram visitados e investigados 21 dos 25 domicílios da comunidade de Quartel do Indaiá, dos quais as condições da moradia e entorno foram levantadas por questionário semi-estruturado contendo questões de estrutura geral (paredes, cobertura, piso, abastecimento de água, esgotamento sanitário), estrutura peridomiciliar (presença de anexos, criação de animais, cultivo de hortaliças), além da composição familiar. A ocorrência de enteroparasitoses foi obtida por meio de consulta aos prontuários de saúde das famílias na Unidade Básica de Saúde responsável pela área. A maioria dos domicílios apresentou infraestrutura composta por paredes de alvenaria, cobertura com telhas de cerâmica sem laje, e o piso principalmente de cimento. Mais da metade das casas não dispunha de banheiros, sendo os dejetos dispensados no peridomicílio. Nas demais os dejetos eram destinados em fossas rudimentares ou no rio. O abastecimento era feito com água não tratada. Todas as casas recebiam luz elétrica. Foi observada ocorrência recorrente de enteroparasitoses, mormente ascaridíase, com frequente eliminação de vermes adultos via oral e pelas fezes, inclusive entre adultos. Apesar de apresentarem estrutura razoável de moradia,os domicílios da comunidade quilombola de Quartel do Indaiá apresentam carências relacionadas ao saneamentoambiental, principalmente no que se refere ao acesso à água tratada e ao destino adequado de dejetos. Tal fato pode serdeterminante na manutenção das enteroparasitoses, as quais têm sido observadas historicamente na comunidade.
\end{abstract}

Palavras-chave: comunidade quilombola; estrutura domiciliar; parasitoses intestinais; Ascaris lumbricoides; contaminação ambiental.

\begin{abstract}
This study aimed to describe structures of domiciles and occurrence of intestinal parasites in a 'quilombola' community at Diamantina municipality, Minas Gerais State. Of a total of 25 domiciles of Quartel do Indaiá, 21 were investigated about housing conditions and surroundings by means of a semi-structured questionnaire about general structure of houses (walls, roof, floor, water supply, sewerage), peridomiciliary structure (presence of outbuildings, animals rearing, vegetable culture), and family composition. Occurrence of intestinal parasites was obtained by search in health records of Basic Health Unit responsible for the area. Most of domiciles presented walls made of adobe or bricks, ceramic covered roofs, without slab, and with floor cement made. More than half of houses had not bathroom, and waste were mainly thrown at peridomicile. In the houses that had bathroom, destiny of waste was cesspool or even the river. Supplied water was not treated. All of the houses had electricity. It was observed continuous occurrence of intestinal parasites, mainly ascariasis, being observed frequently the oral and fecal elimination of adults worms, including among adult patients. Although presenting reasonable housing condition, the domiciles in Quarteldo Indaiá present deficiencies related to environmental sanitation, mainly regarding access to treated water and sewerage. These factors might be determinant in intestinal parasites maintenance, as these infections have been historically found in the community.
\end{abstract}

Keywords: 'quilombola' community; domicile structure, intestinal parasites; Ascarislumbricoides; environmental contamination. 


\section{Introdução}

As parasitoses intestinais ou enteroparasitoses são doenças que apresentam altas incidências, afetando a população humana, sendo um importante problema de saúde pública, principalmente em países em desenvolvimento (SMITH et al., 2001; MACEDO, 2005). Sua presença está associada, quase sempre, ao baixo desenvolvimento econômico, carência de saneamento ambiental e falta de higiene (CARVALHO et al., 2002)

As populações tradicionais ou de interesse especial, como os quilombolas, apresentam deficiências relacionadas ao saneamento ambiental, em especial quanto ao acesso à água tratada, destino de resíduos sólidos e esgotamento sanitário (coleta e tratamento de esgoto), o que contribui com a manutenção de altas prevalências de doenças, inclusive as parasitoses intestinais(CABRALMIRANDA; DATTOLI; DIAS-LIMA, 2010).

$\mathrm{O}$ presente estudo teve como objetivo descrever o contexto ambiental e a ocorrência de enteroparasitoses na comunidade quilombola de Quartel do Indaiá, município de Diamantina, Minas Gerais, permeando aspectos que envolvem a manutenção das enteroparasitoses.

\section{Metodologia}

Este estudo é de caráter descritivo e retrospectivo, tendo sido desenvolvido na comunidade de Quartel do Indaiá, reconhecida como remanescente de quilombo pelo Centro de Documentação Eloy Ferreira da Silva - CEDEFES, estando localizada no município de Diamantina (S 18 $14^{\circ}$; W $43^{\circ} 35^{\prime}$ ), Minas Gerais, na microbacia do Rio Caeté-Mirim, afluente da margem esquerda do Rio Jequitinhonha, a cerca de 40 quilômetros da sede do município, possuindoárea total de 293,5589 hectares (CEDEFES, 2008).

As atividades econômicas que predominavamna comunidade eram o garimpo braçal (faisqueira) e a agricultura de subsistência. Contudo, com a suspensão e proibição dos garimpos, grande parte dos moradores emigrou da localidade em busca de emprego. Sendo assim, 25 famílias permaneceram na localidade, tendo como principal atividade a agricultura familiar, criação de pequenos animais e extrativismo vegetal (MURTA, 2013).

Fizeram parte deste estudo 25 famílias residentes em Quartel do Indaiá, totalizando 126 pessoas, sendo 43 menores de 15 anos, 15 com idade entre 15 e 19 anos, 53 adultos (20 a 59 anos) e 15 idosos (60 anos ou mais).

Para caracterizaçãoda estrutura domiciliar um responsável por cada família foi entrevistado, acompanhando a equipe na observação da casa e do entorno para avaliar a infraestrutura. Para isso, foielaborado e utilizado um questionário constituído de 22 questões: 15 questões fechadas relacionadas ao intradomicílio abordando: o material de construção das paredes, teto, cobertura, 
número de cômodos, cômodos utilizados para dormir, presença de banheiros, destino de dejetos, abastecimento de água, tipo de iluminação, presença de geladeira e fogão; uma pergunta fechada sobre a posse do imóvel; quatro questões fechadas sobre o peridomicílio (presença e tipos de animais domésticos, presença de estruturas de criação de animais, presença de hortas e de árvores frutíferas). Duas questões abertas abordavam, também, a composição familiar dos residentes e a forma de conservação da água de consumo humano. As entrevistas foram conduzidas em outubro de 2011.

A ocorrência de enteroparasitoses em Quartel do Indaiá foi levantada por meio de consulta aos prontuários médicos das famílias assistidas pelo posto de saúde do distrito de São João da Chapada, sede da equipe da Estratégia de Saúde da Família responsável pelo atendimento à comunidade. Nos prontuários foram observadas às informações referentes à idade do paciente, ao diagnóstico, tipo de enteroparasito e medicamento prescrito.

Os dados oriundos dos prontuários e do questionário foram tabulados em planilhas do programa Microsoft Excel® 2003, com posterior análise descritiva.

$\mathrm{O}$ estudo foi realizado respeitando a resolução CNS 196/96, tendo sido aprovado pelo Comitê de Ética em Pesquisa da Pontifícia Universidade Católica de São Paulo - Protocolo 050/2008. A coleta de dados foi conduzida após assinatura do Termo de
Consentimento Livre e Esclarecido pelos participantes.

\section{Resultados e discussão}

3.1 Caracterização da estrutura domiciliar

Das 25 famílias existentes em Quartel do Indaiá, 21 (84,0\%) responderam ao questionário, residindo, nos domicílios abrangidos, 83 pessoas. As demais famílias não participaram desta etapa da pesquisa por não se encontrarem em suas residências no momento da aplicação do questionário.

As informações referentes à infraestrutura dos domicílios são apresentadas na Tabela 1. As paredes eram construídas de alvenaria ou de taipa/adobe revestidas. A maior parte das casas apresentou unicamente o piso de cimento. A média de cômodos por residência foi 6,1 , e o número de cômodos existentes por morador foi de 1,6, o que aponta para uma baixa aglomeração de pessoas. Entretanto, ao analisar o número de cômodos utilizados para dormir em relação ao número de moradores (Tabela 2), observouseaté seis pessoas partilhando o mesmo dormitório e uma média de 1,9 pessoas por dormitório.Alguns trabalhos relacionam que a concentração de pessoas por cômodo como uma medida indireta de condição socioeconômica relacionada à ocorrência de alguns parasitos, como oAscaris lumbricoides(FORTES et al., 2004;ANDRADE et al., 2011) e oStrongyloidesstercoralis(ANDRADE et al., 2011). Além disso, o contato íntimo pode 
favorecer a transmissão deEnterobius vermiculares(BURKHART;BURKHART, 2005) e Giardialamblia (NEWMAN et al., 2001).

Em relação às estruturas sanitárias, mais da metade das casas $(52,4 \%)$ não dispunham de banheiros. A falta de banheiros tem sido observadaem outras comunidades quilombolas, como na comunidade de Tijuaçu, Bahia, onde Cabral-Miranda; Dattoli; Dias-Lima (2010) reportaram ausência de sanitários em $51 \%$ das casas, e na comunidade quilombola Boqueirão também no estado da Bahia, onde Amorim et al. (2013) constataram que $75,3 \%$ das casas não tinham banheiro.

O principal destino dos dejetos em Quartel do Indaiá foi o peridomicílio, o que possibilita a manutenção de contaminação ambiental tanto por ovos de helmintos como por cistos de protozoários, os quais podem permanecer viáveis no solo por até mais de um ano, como no caso do A. lumbricoides (MASSARAet al., 2003). Além disso, tal contaminação viabiliza o desenvolvimento de formas larvais de helmintos cuja via de infecção é transcutânea, como os ancilostomídeos e o S.stercoralis.

A água utilizada para consumo humano na comunidade é proveniente de mananciais, sem receber nenhum tratamento. Em 16 casas $(76,2 \%)$ eram utilizados filtros de barro para a conservação da água, enquanto nas demais a água era conservada em vasilhames de plástico ou consumida diretamente do encanamento. $\mathrm{O}$ consumo de água não tratada pode contribuir para a aquisição de enteroparasitos e comensais, sendo a filtração uma forma de reduzir a contaminação da água (ANDRADE et al., 2011).

Todas as casas apresentavam fogão à lenha, e em sete havia também fogão a gás. Doze casas $(57,1 \%)$ apresentavam televisão com antena parabólica e em 13 (61,9\%) havia geladeira.

Tabela 1 - Características estruturais de domicílios na comunidade quilombola de Quartel do Indaiá, Diamantina, Minas Gerais, $2011(\mathrm{n}=21)$.

\begin{tabular}{|c|c|c|}
\hline Variáveis & Frequência & $\%$ \\
\hline \multicolumn{3}{|l|}{ Tipo de parede } \\
\hline Alvenaria & 12 & 57.1 \\
\hline Taipa/Adobe & 9 & 42.9 \\
\hline \multicolumn{3}{|l|}{ Tipo de cobertura } \\
\hline Cobertura com laje & 1 & 4.8 \\
\hline Cobertura sem laje & 20 & 95.2 \\
\hline \multicolumn{3}{|c|}{ Material da cobertura } \\
\hline Cerâmica & 18 & 85.7 \\
\hline Palha & 2 & 9.5 \\
\hline Cerâmica e amianto & 1 & 4.8 \\
\hline \multicolumn{3}{|l|}{ Piso } \\
\hline Cimento & 13 & 61.9 \\
\hline Terra batida & 6 & 28.6 \\
\hline Cerâmica & 1 & 4.8 \\
\hline Cimento e terra & 1 & 4.8 \\
\hline \multicolumn{3}{|l|}{ Número de cômodos } \\
\hline Três & 1 & 4.8 \\
\hline Quatro & 4 & 19 \\
\hline Cinco & 4 & 19 \\
\hline Seis & 4 & 19 \\
\hline Sete & 3 & 14.3 \\
\hline Oito & 2 & 9.5 \\
\hline Nove & 2 & 9.5 \\
\hline Onze & 1 & 4.8 \\
\hline \multicolumn{3}{|c|}{ Presença de banheiro } \\
\hline Ausente & 11 & 52.4 \\
\hline Presente & 10 & 47.6 \\
\hline \multicolumn{3}{|l|}{ Destino dos dejetos } \\
\hline Fossas rudimentares & 5 & 23.8 \\
\hline Rio Caeté-Mirim & 5 & 23.8 \\
\hline Peridomicílio & 11 & 52.4 \\
\hline \multicolumn{3}{|l|}{ Água canalizada } \\
\hline Intradomicílio & 10 & 47.6 \\
\hline Peridomicílio & 10 & 47.6 \\
\hline Ausente & 1 & 4.8 \\
\hline \multicolumn{3}{|l|}{ Iluminação artificial } \\
\hline Rede pública & 16 & 76.2 \\
\hline Painéis solares & 5 & 23.8 \\
\hline
\end{tabular}

Fonte: Autores. 
O lixo produzido por $18(85,7 \%)$ famílias era queimado em locais em volta das casas, o de duas $(9,6 \%)$ famílias era jogado a céu aberto no peridomicílio e de uma $(4,8 \%)$ era queimado ou jogado a céu aberto no peridomicílio.

Quanto à presença de anexos peridomiciliares, foram observados 14 galinheiros, quatro chiqueiros, sendo que dois não apresentavam porcos no momento da pesquisa, além de seis casas de farinha, dois paióis, um depósito de lenha e uma cobertura anexa com forno de barro.

Quanto aos animais criados no peridomilício, 18 famílias $(85,7 \%)$ criavam galinhas $(\mathrm{n}=297)$, doze $(57,1 \%)$ criavam cachorros $(\mathrm{n}=25)$, seis $(28 \%)$ criavam gatos $(n=12)$, três $(14,3 \%)$ criavam porcos $(n=10)$ e uma família $(4,7 \%)$ criava codornas $(n=5)$ e outra família $(4,7 \%)$, patos $(n=3)$.

Em oito casas (38\%) havia hortas, sendo que uma família tinha uma horta em um terreno central na comunidade com produção destinada ao comércio local e no distrito de São João da Chapada. Os cultivos verificados nas hortas foram alface, beterraba, cebolinha,cenoura, chuchu, couve, maxixe, pepino, pimentão, quiabo e salsa.

O cultivo de legumes e verduras pelos moradores de Quartel do Indaiá em áreas do peridomicílio, associado à falta de saneamento básico que resulta no lançamento dos dejetos humanos diretamente no ambiente, pode contribuir para a dispersão e ocorrência das enteroparasitoses. Alguns autoresapontam a presença de ovos, cistos e larvas de enteroparasitos em diversos tipos de verduras e água contaminada com dejetos humanos (FARIA, et al., 1987; GUILHERMEet al., 1999; TAKAYANAGUI et al., 2001). Dessa forma, o consumo de verduras cruas pode se constituir em uma importante via de transmissão de agentes parasitários (FLORÊNCIO 1986; OLIVEIRA; GERMANO 1992; TAKAYANAGUI et al., 2001).

Tabela 2 - Número de cômodos presentes nas residências e número de cômodos utilizados para dormir na comunidade de Quartel do Indaiá, Diamantina, Minas Gerais

\begin{tabular}{cccc}
\hline Número de casas & $\begin{array}{c}\text { Número de cômodos } \\
\text { utilizados para dormir }\end{array}$ & Número de moradores & $\begin{array}{c}\text { Residentes / cômodos } \\
\text { utilizados para dormir }\end{array}$ \\
\hline 3 & 1 & 1 & 1 \\
2 & 1 & 2 & 2 \\
1 & 1 & 6 & 6 \\
2 & 2 & 2 & 1 \\
2 & 2 & 3 & 1,5 \\
3 & 2 & 4 & 2 \\
2 & 2 & 5 & 2,5 \\
1 & 2 & 7 & 3,5 \\
1 & 3 & 4 & 1,3 \\
2 & 3 & 6 & 2 \\
1 & 4 & 5 & 1,3 \\
1 & 4 & 10 & 2,5 \\
\hline
\end{tabular}

Fonte: Autores. 
3.2 Levantamento da ocorrência de enteroparasitosem prontuários

Os prontuários das famílias da comunidade no período de 1985 a 2011 revelaram a presença tanto de helmintos como de protozoários intestinais:A. lumbricoides, Trichuristrichiura, Ancilostomídeos, G.lamblia,

\section{Entamoebahistolytica/Entamoebadispar,}

Entamoeba coli, Endolimax nana (Tabela 3).Também foi observada a ocorrência deTrichomonasvaginalis,Toxoplasma gondii, alémde mí́ases por'berne' (larvas de Dermatobiahominis).Houve, ainda, suspeita da presença do E. vermiculares pela descrição dos sintomas clínicos: irritabilidade e prurido anal principalmente à noite.

Entre os sintomas que levaram os profissionais de saúde à suspeição de enteroparasitoses e solicitação de exames parasitológicos de fezes ou mesmoà imediata prescrição medicamentosa estavam: náuseas e cefaleia (paciente com oito anos); dor nas pernas e barriga (paciente com quatro anos); cefaleia (paciente com 19 anos); dor de barriga e fezes amolecidas (paciente com um ano); dor epigástrica principalmente à noite $\mathrm{e}$ sudorese (paciente com seis anos); prurido anal durante a noite (paciente com três anos); evacuações com fezes líquidas e avermelhadas (paciente com 72 anos); insônia e nervosismo (seis anos); dor abdominal frequente, abdômen globoso (pacientes com dois e três anos); dor nas pernas e barriga; dor no estômago (paciente com 18 anos); dor de cabeça, dor abdominal, inchaço (paciente com 39 anos); diarreia, cólicas intestinais e náuseas (paciente com nove anos); flatulências, cólicas e diarreia (paciente com 76 anos); bruxismo (paciente com seis anos); linfonodos submandibulares cervicais pré e pós-auriculares enfartados (paciente com sete anos) e também o fato do paciente relatar ficar cuspindo o tempo todo (paciente com 12 anos).

O enteroparasito mais frequentemente assinalado nos prontuários foi o $A$. lumbricoides, detectado em 18 ocasiões.De igual forma, Andrade et al., (2011), na comunidade quilombola de Colônia do Paiol, no estado de Minas Gerais, observaram o predomínio de A. lumbricoides, com positividade de $22,4 \%$ entre 312 exames realizados.

O A. lumbricoides é o parasito que apresenta maior prevalência entre as helmintoses (MACEDO, 2005; CARRILLO; LIMA; NICOLATO, 2005), sobretudo em função da alta produção de ovos pelas fêmeas e resistência destes por mais de um ano no ambiente (MASSARA et al., 2003). A constante eliminação deste parasita por via oral e junto às fezes em Quartel do Indaiá pode estar associada a infecções maciças nessa população.

A maior prevalência de ascaridíase ocorre em crianças acima de um ano de idade, com decréscimo na idade adulta (CROMPTON, 1988), contudo este padrão nem sempre é observado em comunidades 
rurais, onde a prevalência da doença pode não diminuir conforme a faixa etária, o que geralmente acontece em comunidades com alta prevalência e alta carga parasitária, ou mesmo em outras que mantém média prevalência e moradores com carga parasitária baixa (FERREIRA, FERREIRA e NOGUEIRA, 1991). A eliminação de vermes adultos entre moradores da comunidade de Quartel de Indaiá, independentemente da faixa etáriapode, dessa forma, ser indicativo de altas cargas parasitárias, sugerindo, também,alta prevalência deste helminto.
O poliparasitismofoi observado em seis pacientes, sendo que somente um ocorreu sem a presença concomitante de $A$. lumbricoides.

A G. lamblia foi o protozoário mais diagnosticado, sendo encontrado em sete pacientes. Nesse sentido, alguns trabalhos têm demonstrado predominância da G. lamblia sobre os helmintos nos níveis sociais mais favoráveis e tendência inversa em níveis sociais menos favoráveis (FERREIRA; FERREIRA; MONTEIRO, 2000; GIRALDI et al., 2001), o que foi observado em Quartel do Indaiá.

Tabela 3 - Parasitoses ou comensais intestinais entre moradores de Quartel do Indaiá no período de 1985 a 2011 , de acordo com o ano do diagnóstico, a forma de detecção e a idade dos pacientes.

\begin{tabular}{|c|c|c|c|}
\hline Parasito/comensal & Ano & Forma de detecção & Idade \\
\hline T. trichiura & 1985 & Exame de fezes & Não informada \\
\hline Ancilostomídeo & 1985 & Exame de fezes & Não informada \\
\hline A. lumbricoides, ancilostomídeo, T. trichiura & 1985 & Exame de fezes & Não informada \\
\hline T. trichiura, G. lamblia, E. coli & 1985 & Exame de fezes & 56 anos \\
\hline A. lumbricoides, E. histolytica/E. dispar, E. coli & 1996 & Exame de fezes & 73 anos \\
\hline G. lamblia & 2000 & Exame de fezes & 1 ano \\
\hline G. lamblia & 2000 & Exame de fezes & 2 anos \\
\hline A. lumbricoides & 2003 & Eliminação nas fezes & 41 anos \\
\hline A. lumbricoides & 2004 & Eliminação nas fezes & 2 anos \\
\hline A. lumbricoides & 2004 & Eliminação nas fezes & 2 anos \\
\hline A. lumbricoides, G. lamblia, T. trichiura & 2005 & Exame de fezes & 1 ano \\
\hline G. lamblia & 2005 & Exame de fezes & 1 ano \\
\hline G. lamblia & 2005 & Exame de fezes & 27 anos \\
\hline T. trichiura & 2005 & Exame de fezes & 1 ano \\
\hline T. trichiura & 2005 & Exame de fezes & 27 anos \\
\hline A. lumbricoides & 2006 & Eliminação oral & 1 ano \\
\hline A. lumbricoides & 2006 & Eliminação nas fezes & 4 anos \\
\hline A. lumbricoides & 2006 & Eliminação nas fezes & 6 anos \\
\hline A. lumbricoides & 2006 & Eliminação nas fezes & 9 anos \\
\hline A. lumbricoides & 2007 & Eliminação nas fezes & 1 ano \\
\hline A. lumbricoides & 2007 & Eliminação nas fezes & 3 anos \\
\hline A. lumbricoides, Entamoebasp.* & 2007 & $\begin{array}{l}\text { Eliminação nas fezes/ } \\
\text { *Exame de fezes }\end{array}$ & 7 anos \\
\hline A. lumbricoides & 2007 & Eliminação nas fezes & 14 anos \\
\hline G. lamblia & 2007 & Exame de fezes & 5 anos \\
\hline Entamoebasp. & 2007 & Exame de fezes & 35 anos \\
\hline A. lumbricoides & 2008 & Eliminação nas fezes & 2 anos \\
\hline E. histolytica/E. dispar & 2008 & Exame de fezes & 36 anos \\
\hline A. lumbricoides & 2009 & Exame de fezes & 12 anos \\
\hline A. lumbricoides, E. nana, G. lamblia & 2010 & Exame de fezes & 20 anos \\
\hline A. lumbricoides & 2011 & Exame de fezes & 2 anos \\
\hline
\end{tabular}

Fonte: Estratégia de Saúde da Família de São João da Chapada. 
Entre os medicamentos prescritos para o tratamento das enteroparasitoses estavam o metronidazol e o mebendazol, podendo ser receitados juntos ou separados.

Esses medicamentos são rotineiramente utilizados no tratamento de enteroparasitoses, contudo, Sá et al. (2010) alertam para o fato de que o diagnóstico e o tratamento são ações que, quando isoladas, são incapazes de controlar a infecção por agentes enteroparasitários. Segundo esses autores, o retorno das pessoas aos ambientes contaminados contribui para a reinfecção e manutenção das parasitoses o que, de acordo comCHIEFFI et al. 1982 mantém os altos índices de recidiva.

No estudo realizado por Ludwig et al. (1999), observou-se diminuição na prevalência de enteroparasitas na população estudada quando esta passou a ser beneficiada com água tratada e rede de esgoto. No entanto, não adianta apenas disponibilizar saneamento básico adequado para a população, pois a frequência das parasitoses não será alterada se a população não for educada para utilização correta dos recursos disponíveis (FERNANDES; WALMSLEY 1987; SMITH et al., 2001; SÁ et al., 2010).

O presente trabalho apontou a ocorrência histórica de enteroparasitoses, destacando-se a ascaridíase, em uma comunidade quilombola do interior de Minas Gerais. Obviamente, por se tratar de um levantamento de informações coletadas na rotina do serviço de saúde, a prevalência real das parasitoses intestinais provavelmente está subestimada, visto que em muitos casos o diagnóstico foi feito pelo encontro de vermes adultos de $A$. lumbricoides inclusive pela própria população. No caso das outras parasitoses o diagnóstico seria conclusivo por meio do exame parasitológico de fezes, o que possivelmente apontaria uma maior ocorrência dessas na comunidade. Dessa forma, inquéritos parasitológicos posteriores poderão fornecer um panorama mais realista das parasitoses intestinais entre os moradores de Quartel do Indaiá.

\section{Conclusões}

Apesar de apresentarem estrutura razoável de moradia, os domicílios da comunidade quilombola de Quartel do Indaiá apresentam carências relacionadas ao saneamento ambiental, principalmente no que se refere ao acesso à água tratada e ao destino adequado de dejetos. Tal fato pode ser determinante na manutenção das enteroparasitoses, as quais têm sido observadas historicamente na comunidade.

Para que ocorra redução das parasitoses intestinais na comunidade de Quartel do Indaiá é necessária a melhoria das condições de saneamento ambiental, com construção de estruturas sanitárias (banheiros, fossas sépticas, rede de esgoto), tratamento da água de consumo humano, alémde ações de educação em saúde visando à promoção do 
autocuidado e consequente prevenção de novas infecções na população.

\section{Referências}

ANDRADE, E. C.et al.Prevalência de parasitoses intestinais em comunidade quilombola no Município de Bias Fortes, Estado de Minas Gerais, Brasil, 2008. Epidemiologia e Serviços de Saúde, v. 20, n. 3, p. 337-344, 2011.

AMORIM, M. M. et al.Avaliação das condições sanitárias e de saúde da comunidade quilombola Boqueirão, Bahia, Brasil. Bioscience Journal, v. 29, n. 4, p. 1049-1057, 2013.

BURKHART, C.N.; BURKHART, G.N. Assessment of frequency, transmission, and genitourinary complications of enterobiasis (pinworms). International Journal of Dermatology, v. 44, n. 10, p. 837840, 2005.

CABRAL-MIRANDA, G.; DATTOLI, V. C. C.; DIAS-LIMA, A. Enteroparasitos e condições socioeconômicas e sanitárias em uma comunidade quilombola semiárido baiano. Revista de Patologia Tropical. v. 39, n. 1, p. 48-55, 2010.

CARRILLO, M. R. G.; LIMA, A. A.; NICOLATO, R. L. C. Prevalência de enteroparasitoses em escolares do bairro Morro de Santana em Município de Ouro Preto, MG. Revista Brasileira de Análises Clínicas, v. 37, n. 3, p. 191-193, 2005.

CARVALHO, O. S. et al. Prevalência de helmintos intestinais em três mesoregiões do estado de Minas Gerais. Revista da Sociedade Brasileira de Medicina Tropical, v. 35, n. 6, p. 597-600, 2002.

CEDEFES. Comunidades quilombolas de

Minas Gerais no séc. XXI - História e resistência / organizado por Centro de Documentação Eloy Ferreira Silva. Belo Horizonte: AUTÊNTICA/CEDEFES, 2008.
CHIEFFI, P. P. et al. Aspectos epidemiológicos das enteroparasitoses no Estado de São Paulo, Brasil.Revista da Sociedade Brasileira de Medicina, v. 99, p. 34-36, 1982.

CROMPTON, D.W.T. The prevalence of ascariasis.Parasitology Today, v. 4, n. 6, p. 162-169, 1988.

FARIA, J. A. S. et al. Estudo de alguns aspectos de disseminação de enteroparasitas na cidade de Salvador/Bahia: estudo da poluição de água de irrigação de hortas por cistos e ovos de enteroparasitas.Revista Bahiana de Saúde Pública, v. 13/14, n. 4/1 p. 141-144, 1987.

FERNANDES, E. C.; WALMSLEY, R. Parasitoses intestinais: uma visão popular. Revista do Instituto Materno Infantil de Pernambuco, v. 1, n. 2, p. 145-149, 1987.

FERREIRA, C. S.; FERREIRA, M. U.; NOGUEIRA, M. R. Prevalência e intensidade de infecção por Ascarislumbricoides em amostra populacional urbana (São Paulo, SP). Cadernos de Saúde Pública, v. 7, n. 1, p. 8289, 1991.

FERREIRA, M. U.; FERREIRA, C. S.; MONTEIRO, C. A. Tendência secular das parasitoses intestinais na infância na cidade de São Paulo (1984-1996). Revista de Saúde Pública, v. 37, n. 6, p. 73-82, 2000.

FLORÊNCIO, M. L. Q. Estudo de alguns aspectos epidemiológicos das enteroparasitoses em famílias da cidade de Pradópolis, São Paulo. Jornal de Pediatria, v. 60, n. 6, p. 291-296, 1986.

FORTES, B. P. M. D. et al. Modelagem geoestatística da infecção por Ascaris lumbricoides. Cadernos de Saúde Pública, v. 20, n. 3, p. 727-734, 2004.

GIRALDI, N.et al. Prevalência de enteroparasitoses em crianças de creches e alunos de rede municipal de ensino de Rolândia, Paraná. Revista da Sociedade 
Brasileira de Medicina Tropical, v. 34, n. 4, p. 385-387, 2001.

GUILHERME, A. L. F.et al. Prevalência de enteroparasitas em horticultores e hortaliças da Feira do Produtor de Maringá, Paraná.

Revista da Sociedade Brasileira de

Medicina Tropical, v. 32, n. 4, p. 405-411, 1999.

LUDWIG, K. M. et al. Correlação entre condições de saneamento básico e parasitoses intestinais na população de Assis, Estado de São Paulo. Revista da Sociedade Brasileira de Medicina Tropical, v. 32, n. 5, p. 547555, 1999.

MACEDO, H. S. Prevalência de parasitos e comensais intestinais em crianças de escolas da rede pública municipal de Paracatu (MG). Revista Brasileira de Análises Clínicas, v. 37, n. 4, p. 209-213, 2005.

MASSARA, C. L. et al. Atividade de detergentes e desinfetantes sobre a evolução dos ovos de Ascaris lumbricoides. Cadernos de Saúde Pública, v. 19, n. 1, p. 335-340, 2003.

MURTA, N.M.G. O acaso dos casos: estudos sobre alimentação-nutrição, cultura e história. Tese de doutorado. Programa de Estudos Pós-graduados em Ciências Sociais. Pontifícia Universidade Católica de São Paulo. São Paulo, 2013. 171 pp.

NEWMAN, R. D. et al. A longitudinal study of Giardia duodenalis infection in north-east Brazilian children.Tropical Medicine andInternational Health, v. 6, n. 8, p. 624634, 2001.

OLIVEIRA, C.A.F.; GERMANO, P.M.L. Estudo da ocorrência de enteroparasitas em hortaliças comercializadas na região metropolitana de São Paulo, SP, Brasil. I Pesquisa de helmintos. Revista de Saúde Pública, v. 26, n. 4, p. 283-289, 1992.

SÁ, S. R. et al. Enteroparasitoses em Crianças Assistidas pela Estratégia Saúde da Família. In: Ribeiro, M.; Ribeiro, L.C.C. In: Pesquisas \& Vivências na Atenção Primária à Saúde. Diamantina: FUNDAEPE, v. 1, p. 309-318, 2010.

SMITH, H. M. et al. Prevalence and intensity of infections of Ascarislumbricoides and Trichuristrichiura and associated socio demographic variables in four rural Honduran communities.Memórias do Instituto

Oswaldo Cruz, v. 96, n. 3, p. 303-314, 2001.

TAKAYANAGUI, O. M. et al.Fiscalização de verduras comercializadas no município de Ribeirão Preto, SP. Revista da Sociedade Brasileira de Medicina Tropical, v. 34, n. 1, p. 37-41, 2001. 